Louisiana State University

LSU Digital Commons

6-1-2009

\title{
Risk of Secondary Malignant Neoplasms From Proton Therapy and Intensity-Modulated X-Ray Therapy for Early-Stage Prostate Cancer
}

Jonas D. Fontenot

University of Texas MD Anderson Cancer Center

Andrew K. Lee

University of Texas MD Anderson Cancer Center

Wayne D. Newhauser

University of Texas MD Anderson Cancer Center

Follow this and additional works at: https://digitalcommons.Isu.edu/physics_astronomy_pubs

\section{Recommended Citation}

Fontenot, J., Lee, A., \& Newhauser, W. (2009). Risk of Secondary Malignant Neoplasms From Proton Therapy and Intensity-Modulated X-Ray Therapy for Early-Stage Prostate Cancer. International Journal of Radiation Oncology Biology Physics, 74 (2), 616-622. https://doi.org/10.1016/j.ijrobp.2009.01.001

This Article is brought to you for free and open access by the Department of Physics \& Astronomy at LSU Digital Commons. It has been accepted for inclusion in Faculty Publications by an authorized administrator of LSU Digital Commons. For more information, please contact ir@lsu.edu. 


\title{
RISK OF SECONDARY MILIGNANT NEOPLASMS FROM PROTON THERAPY AND INTENSITY-MODULATED X-RAY THERAPY FOR EARLY-STAGE PROSTATE CANCER
}

\author{
Jonas D. Fontenot, Ph.D. ${ }^{\star}{ }^{\dagger}$, Andrew K. Lee, M.D., M.P.H. ${ }^{\ddagger}$, and Wayne D. Newhauser, Ph.D. \\ *Department of Radiation Physics The University of Texas M. D. Anderson Cancer Center, \\ Houston, TX \\ FDepartment of Radiation Oncology, The University of Texas M. D. Anderson Cancer Center, \\ Houston, TX
}

\begin{abstract}
Purpose-To assess the risk of a secondary malignant neoplasm (SMN) from proton therapy relative to intensity-modulated radiation therapy (IMRT) using X-rays, taking into account contributions from both primary and secondary sources of radiation, for prostate cancer.
\end{abstract}

Methods and Materials-A proton therapy plan and a 6-MV IMRT plan were constructed for 3 patients with early-stage adenocarcinoma of the prostate. Doses from the primary fields delivered to organs at risk of developing an SMN were determined from treatment plans. Secondary doses from the proton therapy and IMRT were determined from Monte Carlo simulations and available measured data, respectively. The risk of an SMN was estimated from primary and secondary doses on an organ-by-organ basis by use of risk models from the Committee on the Biological Effects of Ionizing Radiation.

Results-Proton therapy reduced the risk of an SMN by $26 \%$ to $39 \%$ compared with IMRT. The risk of an SMN for both modalities was greatest in the in-field organs. However, the risks from the in-field organs were considerably lower with the proton therapy plan than with the IMRT plan. This reduction was attributed to the substantial sparing of the rectum and bladder from exposure to the therapeutic beam by the proton therapy plan.

Conclusions-When considering exposure to primary and secondary radiation, proton therapy can reduce the risk of an SMN in prostate patients compared with contemporary IMRT.

\section{Keywords}

Proton therapy; Intensity-modulated X-ray therapy; Prostate cancer; Second cancers

\footnotetext{
Copyright @ 2009 Elsevier Inc. Printed in the USA. All rights reserved

Reprint requests to: Wayne Newhauser, Ph.D., Department of Radiation Physics, University of Texas M. D. Anderson Cancer Center, 1515 Holcombe Boulevard Unit 94, Houston, TX 77030. Tel: (713) 563-1520; Fax: (713) 563-2545; wnewhaus@mdanderson.org. †Present address: Department of Medical Physics, Mary Bird Perkins Cancer Center, Baton Rouge, LA Conflict of interest: none.
} 


\section{INTRODUCTION}

Long-term survivors of prostate cancer who underwent radiation therapy face a small but significant risk of radiation-induced secondary malignant neoplasm (SMN) developing as a result of their exposure to ionizing radiation (1-3). Many of these radiation-induced SMNs arise in normal tissues immediately adjacent to the target volume (2), where tissues are exposed to high radiation doses from the primary beam. In addition, SMNs also appear in tissues well outside the radiation field (2) and are associated with exposure to low doses of scattered and leakage radiation attributable to imperfections in radiation delivery devices (4). On the basis of these observations, it appears that contributions from primary (therapeutic) and secondary (i.e., scatter and leakage) radiation should be taken into account when assessing the risk of second cancers after external beam radiation therapy.

Because of their sharp characteristic Bragg peak, proton beams are ideally suited for treating deep-seated tumors, such as in the prostate. Recent treatment planning studies have shown proton therapy's potential for significantly reducing the integral dose required to deliver the prescribed treatment compared with contemporary intensity modulated radiation therapy (IMRT) using X-rays $(5,6)$. One proposed benefit of the increased dose sparing offered by proton therapy is a reduction in the incidence of radiogenic second cancers. Recently, Schneider et al. $(7,8)$ estimated that the incidence of second cancers after prostate irradiation could be reduced by a factor of two using spot-scanned proton therapy compared with IMRT. However, most proton centers currently in operation today use passively scattered delivery techniques (9), for which leakage neutrons have become a concern (10, 11). The high relative biologic effectiveness (RBE) of neutrons for carcinogenesis may substantially increase the incidence of SMNs. Unfortunately, until now, no studies have attempted to assess the theoretic reduction in SMNs offered by the tissue sparing of passively scattered proton therapy against the purported increase in SMNs due to secondary neutrons.

The goal of this study was to assess the risk of an SMN after proton therapy of the prostate compared with the risk after 6-MV IMRT for a typical patient, taking into account contributions from both primary and secondary radiation. We determined primary doses from dose-volume histograms of treatment plans constructed with a commercial planning system. We then determined secondary doses from proton therapy using Monte Carlo simulations and compared them with secondary doses from IMRT taken from the literature.

\section{METHODS AND MATERIALS}

\section{Organs at risk}

We assessed the risk of an SMN after radiation therapy of the prostate for 3 patients with varying anatomic separation (determined by the required proton beam range) treated at our institution: a 61-year-old man with T2a adenocarcinoma (small separation), a 56-year-old man with T2a adenocarcinoma (medium separation), and a 47-year-old man with T1c adenocarcinoma (large separation). Risk of an SMN was quantified by examining radiation doses to at-risk organs during treatment. At-risk organs were defined as those at highest risk of developing a radiation-induced SMN, as specified by Publication 60 of the International 
Commission on Radiological Protection (ICRP) (12). These organs include the bladder, colon, lungs, stomach, bladder, liver, esophagus, thyroid, bone marrow, skin, bone surface, and remainder (i.e., all other tissues). The dose to the colon was taken as the mass-weighted mean of the doses to the colon and rectum.

\section{Primary dose}

The principal source of radiation exposure in normal tissues during radiation therapy for prostate cancer is the primary radiation field. Exposures of organs of risk to the primary field were estimated from treatment plans, which were constructed by use of a commercial treatment planning system (Eclipse, version 8.0; Varian Medical Systems, Palo Alto, CA). Two treatment approaches were assessed: a passively scattered proton technique and 6-MV IMRT technique. Water-filled rectal balloons were used as part of both plans to improve rectal sparing. Planning approaches and dose constraints used for optimization were those used clinically at M. D. Anderson Cancer Center, Houston, TX (13).

The 6-MV IMRT treatment was planned to deliver $75.6 \mathrm{~Gy}$ in 42 fractions to the planning target volume, which consisted of a 7-mm uniform margin (with the exception of $5 \mathrm{~mm}$ in the posterior direction) surrounding the prostate and proximal seminal vesicles, or clinical target volume (CTV). The treatment was planned by use of the step-and-shoot delivery technique on a Varian Clinac 2100 system (Varian Medical Systems) and was constructed by use of an eight-field co-planar beam arrangement. The gantry angles for the fields were $30^{\circ}, 65^{\circ}, 100^{\circ}, 135^{\circ}, 225^{\circ}, 260^{\circ}, 295^{\circ}$, and $330^{\circ}$. A total of 691,664 , and 739 monitor units were required to deliver each fraction for the small, medium, and large patient, respectively.

The proton treatment was planned to deliver 75.6 cobalt gray equivalent (CGE) (i.e., 68.7 Gy $\times 1.1 \mathrm{RBE}$ ) in 42 fractions, with distal and lateral margins of $1.2 \mathrm{~cm}$ around the CTV. The proton therapy plan was constructed to deliver the treatment by use of the synchrotronbased, passively scattered beam line (14) at the University of Texas M. D. Anderson Cancer Center's proton therapy center, and it used the parallel-opposed, lateral two-field orientation described by Slater $e t$ al. (15). The required beam range for the small, medium, and large patient was 22.1,24.6, and $27.0 \mathrm{~cm}$, respectively, in water, which corresponded to a proton beam energy of 225 to $250 \mathrm{MeV}$ from the synchrotron and the insertion of 1 to $4 \mathrm{~cm}$ of water-equivalent range shifter material in the treatment head. The required width of the spread-out Bragg peak was $10 \mathrm{~cm}$ for all 3 patients. Patient-specific devices-including a milled brass collimator and a polymethyl methacrylate range compensator-were used to conform the proton dose to the target and were contained in an $18 \times 18-\mathrm{cm}^{2}$ snout.

After the plans were completed, absorbed doses from the primary field in the adjacent organs at risk (namely the rectum and bladder) were taken from dose-volume histograms calculated by the treatment planning system.

\section{Secondary dose}

Normal tissues are also exposed to secondary radiation doses during the course of treatment. Exposures of organs at risk to secondary radiation were estimated by use of Monte Carlo 
simulations for the proton treatment and from measured data available in the literature for the IMRT treatment.

Proton therapy-Secondary doses from proton therapy were simulated by Monte Carlo methods, which use random numbers to simulate the stochastic process of radiation transport in matter. The treatment plan was imported into a Monte Carlo model of the M. D. Anderson passive scattering beam line via in-house software (16). The patient was modeled as a stylized anthropomorphic phantom following the methods of Fontenot et al. (17). Inside the phantom, absorbed dose from secondary radiation (neutrons and photons) was calculated in spherical receptors inside organs at risk of an SMN. To account for the increased carcinogenic risk of neutrons, absorbed dose from neutrons in each organ, $D_{\mathrm{T}}$, was multiplied by a radiation weighting factor, $w_{\mathrm{R}}$, such that

$$
H_{\mathrm{T}}=D_{\mathrm{T}} \times w_{\mathrm{R}}
$$

where $H_{\mathrm{T}}$ is the equivalent dose. The values of $w_{\mathrm{R}}$ were derived from recommendations in Report 92 of the ICRP by use of methods described by Zheng et al. (18). The resulting equivalent dose in each organ (reported in sieverts per gray for treatment) was scaled by the prescription dose to provide a total equivalent dose in each organ for the entire treatment course. Additional details of the simulation procedure can be found elsewhere (17).

Intensity-modulated X-ray therapy-Secondary doses from IMRT are relatively well understood and have received considerable attention in the literature. Organ doses from secondary radiation during IMRT depend on electron beam energy, distance of the organ from the irradiated volume, depth in the patient, linear accelerator manufacturer, and other factors (4). At lower energies ( $\$ 8 \mathrm{MV}$ ), secondary radiation is composed only of scattered and leakage photon radiation. Near the field, photons scattered in the patient predominate. Further from the field, leakage photons from the treatment head predominate $(19,20)$. At higher energies (>8 MV), in addition to photon scatter and leakage, photoneutrons are generated in the primary collimator, jaws, electron target, and flattening filter. Because of the high RBE of neutrons and because of the large monitor unit requirement of IMRT, energies of $18 \mathrm{MV}$ and above are not generally used for IMRT treatments, especially in younger patients.

In this work secondary doses from 6-MV IMRT of the prostate were estimated from data reported by Kry et al. (4) and Howell et al. (21) for Varian accelerators (Varian Medical Systems). In those studies scattered and leakage photons were measured in an anthropomorphic phantom using thermoluminescent dosimeters for 5- and 7-field step-andshoot IMRT prostate plans. The available data were fit with an exponential curve such that the stray photon dose could be computed for the exact locations of the sensitive organs in the proton therapy phantom. The fit to the data was given by

$$
y=1.02 \times 10^{-4} \cdot \operatorname{Exp}^{-0.117 \cdot x}+1.95 \times 10^{-6} \cdot \operatorname{Exp}^{-5.44 \times 10^{-11}} \cdot x
$$

where $y$ is the secondary dose (in sieverts per monitor unit) at a lateral distance $x$ from the isocenter. 
The stray dose (in sieverts per monitor unit) determined for each organ was scaled by the total number of monitor units required to deliver the planned IMRT treatment $(691,664$, and 739 per fraction for the small, medium, and large patient, respectively).

\section{Risk models}

In this study the quantity ratio of excess relative risk $(R R R)$ was used to quantify the risk of an SMN after proton therapy relative to IMRT. The $R R R$ was defined as

$$
R R R=E R R_{\text {proton }} / E R R_{I M R T}
$$

where $E R R$ denotes the excess relative risk of a radiation-induced cancer developing after exposure to proton therapy ( $\left.E R R_{\text {proton }}\right)$ or IMRT ( $\left.E R R_{\mathrm{IMRT}}\right)$. The $E R R$ is a radiation epidemiologic quantity used to describe the rate of cancer incidence in a radiation-exposed population relative to an unexposed population. For each modality (proton therapy or IMRT), the $E R R$ ( $\left.E R R_{\text {modality }}\right)$ was calculated as

$$
E R R_{\text {modality }}=\left(\sum_{\mathrm{T}} E R R_{\mathrm{T}}\right)_{\text {modality }}
$$

where $E R R_{\mathrm{T}}$ is the ERR of a radiation-induced cancer developing in tissue $T$. For each tissue, the ERR was given by

$$
E R R_{\mathrm{T}}=H_{\mathrm{T}} \times R_{\mathrm{T}}
$$

where $H_{\mathrm{T}}$ is equivalent dose and $R_{\mathrm{T}}$ is an organ-specific risk coefficient. For this study, organ-specific risk coefficients were taken from a recent report of the Committee on the Biological Effects of Ionizing Radiation (BEIR) (22), issued by the National Academy of Sciences. The BEIR VII Committee comprehensively evaluated available data on the incidence of cancer resulting from exposure to low levels of ionizing radiation. Much of the data regarding radiation-induced malignancies were drawn from survivors of the Japanese atomic bomb, although the committee also incorporated data from various in vitro, animal, and medical radiation studies. The BEIR Committee concluded that there was a linear-no threshold (LNT) relationship between cancer risk and radiation exposure at low doses $(<2.5$ Sv). The BEIR VII risk coefficients, adjusted for sex and age of the patient, are listed in Table 1. The risk of leukemia was calculated based on the dose to bone marrow, estimated as the mean dose for all organs. Any organs for which coefficients were not explicitly provided were considered to be "other solid cancers."

Risk coefficients in the BEIR VII report are provided for cases of uniform radiation exposure at low doses. However, tissues near the irradiated volume (the rectum and bladder in the case of prostate cancer) are partially exposed to high doses. In these cases, for simplicity, the LNT model was linearly extrapolated to high doses, and $E R R_{\mathrm{T}}$ was calculated voxel by voxel as 


$$
E R R_{\mathrm{T}}=\frac{1}{\mathrm{~N}} \sum_{\mathrm{i}=1}^{\mathrm{N}}\left[H_{\mathrm{T}_{\mathrm{i}}} \times R_{\mathrm{T}}\right]
$$

where $H_{\mathrm{T}_{\mathrm{i}}}$ is the equivalent dose in the ith voxel, $R_{T}$ is the organ-specific risk coefficient at dose $H_{\mathrm{T}}$ computed from the BEIR VII report, and $N$ is the total number of voxels.

Baseline risk calculations used the LNT model to relate cancer risk to an equivalent dose in each organ. For organs far from the radiation field, which are exposed to relatively low equivalent doses, this approximation is valid. However, during radiation therapy of the prostate, the rectum and bladder are exposed to high equivalent doses, where the shape of the risk model is not known. Although the baseline calculations used the LNT model, there are plausible alternatives, such as the linear-exponential and linear-plateau models, which describe the dose-response relationship at high doses (23). Therefore, to test the sensitivity of our analysis to changes in the risk model, we calculated the RRR using four additional risk models, each consistent with LNT at low doses but with a different shape at high doses to bound the interval of plausible risks. Figure 1 shows the additional risk models that were examined. For the linear-exponential and linear-plateau models, the risk rolled off at low equivalent dose $(10 \mathrm{~Sv})$ or high equivalent dose $(40 \mathrm{~Sv})$.

\section{RESULTS}

Both plans provided adequate coverage of the CTV and margins (Fig. 2), with the proton therapy plan providing slightly greater dose uniformity within the CTV. The minimum, mean, and maximum doses to the CTV from the proton therapy plan were $76.5 \pm 0.2 \mathrm{CGE}$, $78.0 \pm 0.1 \mathrm{CGE}$, and 79.2 $\pm 0.3 \mathrm{CGE}$, respectively. For the IMRT plan, the corresponding values were similar, at $77.6 \pm 0.7 \mathrm{~Gy}, 79.2 \pm 0.4 \mathrm{~Gy}$, and $80.9 \pm 0.3 \mathrm{~Gy}$, respectively.

Both plans provided acceptably low doses to the surrounding critical structures; however, the proton therapy plan provided substantially lower doses at low and intermediate levels in the bladder and rectum (Fig. 3). The mean and maximum doses to the bladder from the proton therapy plan were 13.2 $\pm 4.1 \mathrm{CGE}$ and $78.5 \pm 0.2 \mathrm{CGE}$, respectively, compared with $22.2 \pm 3.9$ Gy and $80.6 \pm 0.3 \mathrm{~Gy}$, respectively, from the IMRT plan. The mean and maximum doses to the rectum from the proton therapy plan were 20.8 $\pm 1.4 \mathrm{CGE}$ and $78.5 \pm$ 0.3 CGE, respectively, compared with $28.1 \pm 1.9$ Gy and $79.8 \pm 0.4 \mathrm{~Gy}$, respectively, from the IMRT plan.

In general, secondary doses from proton therapy were smaller than secondary doses from IMRT near the field but were greater for proton therapy than IMRT in tissue far from the therapeutic fields (Fig. 4). Secondary doses outside the radiation field were highest in organs closest to the irradiated volume and decreased with increasing distance from the isocenter. Scattered doses from the IMRT treatment were as high as $2.5 \mathrm{~Sv}$ near the irradiated volume (rectum) and as low as $61 \mathrm{mSv}$ (esophagus/thyroid) far from the treatment fields. Similar to IMRT, secondary doses from proton therapy were highest near the irradiated volume (up to $1 \mathrm{~Sv}$ ) and decreased with increasing distance from the isocenter (as low as $114 \mathrm{mSv}$ ). Leakage neutrons were the predominant source of out-of-field exposures for the proton 
therapy plan. Most of these neutrons were produced in the treatment unit, although organs near the radiation field were exposed to a substantial equivalent dose from neutrons created within the patient (17).

The baseline RRR calculations (assuming the LNT model) for the patients examined in this study are shown in Table 1. For both modalities, the risk of an SMN was predominated by the in-field organs, defined as tissues completely or partially irradiated by the primary field (i.e., colon/rectum and bladder). By use of the LNT model, in-field organs accounted for approximately $90 \%$ of the total ERR for proton therapy and IMRT. Of the two in-field organs, the bladder was the dominant source of elevated risk. The bone marrow was the dominant source of elevated risk in the out-of-field organs for both modalities. The calculated $R R R$ s for the small, medium, and large patients were $0.61,0.66$, and 0.74 , respectively, suggesting that the proton therapy plan could reduce the risk of an SMN by $39 \%, 34 \%$, and $26 \%$, respectively, compared with IMRT.

In general, the calculated $R R R$ showed only a small sensitivity to dose-response model (Fig. 5). For all risk models, the primary field was the primary contributor to the ERR. For the medium patient, the calculated reduction in the risk of an SMN after proton therapy compared with IMRT ranged from $32 \%$ to $40 \%$ for all risk models examined. The primary reason for this reduction was the ability of proton therapy to spare the bladder and rectum from exposure to therapeutic radiation more effectively than IMRT. Although the $R R R$ were not sensitive to changes in the dose-response model, the ratio of in-field SMNs to out-offield SMNs was sensitive to these changes. Whereas the in-field organs accounted for about $90 \%$ of the ERR for both modalities by use of the LNT model, this value fell to just over $50 \%$ when we used the linear-exponential model rolling off at $10 \mathrm{~Sv}$. This result follows from the fact that the out-of-field $E R R$ constitutes a greater proportion of the total $E R R$ when a risk model that suppresses risk in high-dose regions is used.

\section{DISCUSSION}

We estimated the risk of a radiation-induced SMN after proton therapy compared with 6MV IMRT for prostate cancer, taking into account primary and secondary radiation exposures. The results of this study indicate that passively scattered proton therapy-even taking into account stray neutron exposures - can reduce the incidence of SMNs in a population of prostate patients compared with IMRT. Because the primary beam predominated the second cancer risk for both modalities, the impact of stray neutrons from proton therapy appears to be of secondary importance. However, these results also suggest that reducing stray radiation exposures—either through the use of beam scanning or with local shielding techniques (24)—can lower the risk of SMNs.

These results are significant for a number of reasons. First, the prevalence of prostate cancer (217,000 new cases diagnosed in the United States in 2007) means that even a low incidence of radiogenic second malignancies would correspond to a considerable number of men in whom SMNs develop. Furthermore, most patients currently being treated with proton beams are treated for prostate disease. Finally, the finding that second cancer risks associated with proton therapy of the prostate are predominated by the primary beam, and not stray neutron 
radiation, is particularly noteworthy, given the considerable debate that has surrounded the production of stray neutrons during proton therapy $(10,11,25)$.

Our findings are quantitatively and qualitatively similar to those of previous studies. This study found that using proton beams to treat prostate cancer can significantly reduce the dose received by adjacent critical structures (i.e., the rectum and bladder) compared with IMRT. Similar findings have been reported in treatment planning studies of proton therapy for treating prostate cancer $(5,6,26)$. In the study most closely related to this work, Schneider et al. (7) estimated that the risk of a radiation-induced second cancer after spotscanned proton therapy for prostate cancer, relative to 6-MV IMRT, was approximately 0.50. To compare the predictions of Schneider et al. with our findings, we may adjust our estimates to approximate the risk of an SMN from a scanned-beam treatment. On the basis of the assumption that stray neutrons emanating from the nozzle during spot-scanned proton therapy are considered negligible, the internal neutron component (i.e., neutrons created within the patient) reported by Fontenot et al. (17) can be used to estimate the risk of second cancer from spot scanning. With that approach, the estimated $R R R$ for a spot-scanned proton beam from this work was 0.56 . Given the differences in methods used for risk calculations in this work and that of Schneider et al. [BEIR VII risk models vs. organ-equivalent dose models (27), intensity-modulated proton therapy vs. spot scanning, and so on], the relative risk estimates agree remarkably well. This agreement lends confidence to the major findings of this work.

The methods used in this work provide the most rigorous and comprehensive estimates of radiation doses from proton radiotherapy of the prostate. Rather than approximate secondary dose in air or in simple water phantoms $(18,28,29)$, this study used data from a detailed computerized anthropomorphic phantom, with secondary radiation exposures determined on an organ-by-organ basis. Because stray radiation from proton therapy has been shown to be sensitive to a number of variables $(17,18,24)$, patient-specific machine settings were implemented in our Monte Carlo model.

This work also had several limitations. First, radiation weighting factors for neutrons were derived from ICRP Report 92. There are considerable uncertainties associated with these values $(11,30)$. The findings of this work may, in theory, be different if the radiation weighting factor of neutrons were found to be considerably greater than currently believed. Second, our study was limited to an examination of proton therapy and IMRT for prostate cancer. Although these modalities are the two most common forms of external beam therapy for prostate cancer, there are other emerging external beam radiation therapy technologies of interest, including TomoTherapy (TomoTherapy Inc., Madison, WI), Cyberknife (Accuray Inc., Sunnyvale, CA), and RapidArc (Varian Medical Systems). These technologies warrant further investigation from the standpoint of second cancer induction. Finally, rectal balloons were used in both the proton therapy and IMRT plans to displace the rectum (thus improving rectal sparing) and preserve anatomic parity between the two treatment approaches.

Although rectal balloons are routinely used in proton therapy to spare the rectum, they are not typically used for IMRT treatment. Thus, in practice, the true ERR from IMRT may be greater than the present calculations, resulting in greater benefit from proton therapy. 


\section{CONCLUSIONS}

Despite the presence of secondary neutrons, proton therapy can reduce the calculated incidence of radiogenic second cancers in prostate patients compared with IMRT, for both the passively scattered and scanned proton beams considered, as well as for a wide variety of dose-response models. This finding was principally attributed to the ability of proton therapy to reduce the primary dose in sensitive organs near the target volume compared with IMRT.

\section{Acknowledgments}

We thank Drs. Charles Bloch, David Followill, Uwe Titt, Ming Zhang, Phillip Taddei, and Dragan Mirkovic for their contributions to this work.

This work was supported in part by Northern Illinois University through a subcontract of Department of Defense Contract W81XWH-08-1-0205.

\section{REFERENCES}

1. Baxter NN, Tepper JE, Durham SB, et al. Increased risk of rectal cancer after prostate radiation: A population-based study. Gastroenterology. 2005; 128:819-824. [PubMed: 15825064]

2. Brenner DJ, Curtis RE, Hall EJ, et al. Second malignancies in prostate carcinoma patients after radiotherapy compared with surgery. Cancer. 2000; 88:398-406. [PubMed: 10640974]

3. Moon K, Stukenborg GJ, Keim J, et al. Cancer incidence after localized therapy for prostate cancer. Cancer. 2006; 107:991-998. [PubMed: 16878323]

4. Kry SF, Salehpour M, Followill DS, et al. Out-of-field photon and neutron dose equivalents from step-and-shoot intensity-modulated radiation therapy. Int J Radiat Oncol Biol Phys. 2005; 62:12041216. [PubMed: 15990026]

5. Mock U, Georg D, Bogner J, et al. Treatment planning comparison of conventional, 3D conformal, and intensity-modulated photon (IMRT) and proton therapy for paranasal sinus carcinoma. Int $\mathbf{J}$ Radiat Oncol Biol Phys. 2004; 58:147-154. [PubMed: 14697432]

6. Trofimov A, Nguyen PL, Coen JJ, et al. Radiotherapy treatment of early-stage prostate cancer with IMRT and protons: A treatment planning comparison. Int J Radiat Oncol Biol Phys. 2007; 69:444453. [PubMed: 17513063]

7. Schneider U, Lomax A, Besserer J, et al. The impact of dose escalation on secondary cancer risk after radiotherapy of prostate cancer. Int J Radiat Oncol Biol Phys. 2007; 68:892-897. [PubMed: 17459608]

8. Schneider U, Lomax A, Pemler P, et al. The impact of IMRT and proton radiotherapy on secondary cancer incidence. Strahlenther Onkol. 2006; 182:647-652. [PubMed: 17072522]

9. Koehler A, Schneider R, Sisterson J. Flattening of proton dose distributions for large-field radiotherapy. Med Phys. 1977; 4:297-301. [PubMed: 407436]

10. Hall EJ. Intensity-modulated radiation therapy, protons, and the risk of second cancers. Int J Radiat Oncol Biol Phys. 2006; 65:1-7. [PubMed: 16618572]

11. Hall EJ. The impact of protons on the incidence of second malignancies in radiotherapy. Technol Cancer Res Treat. 2007; 6:31-34. [PubMed: 17668949]

12. International Commission on Radiological Protection. 1990 Recommendations of the International Commission on Radiological Protection. Ann ICRP. 1991; 21:1-201.

13. Zhang X, Dong L, Lee AK, et al. Effect of anatomic motion on proton therapy dose distributions in prostate cancer treatment. Int J Radiat Oncol Biol Phys. 2007; 67:620-629. [PubMed: 17236979]

14. Smith, A.; Newhauser, W.; Latinkic, M., et al. The University of Texas MD Anderson Cancer Center Proton Therapy Center. In: Duggan, J.; Morgan, I., editors. 17th International Conference on the Application of Accelerators in Research and Industry; Denton, TX: American Institute of Physics. 2002. p. 1073-1076. 
15. Slater JD, Yonemoto LT, Rossi CJ Jr. et al. Conformal proton therapy for prostate carcinoma. Int J Radiat Oncol Biol Phys. 1998; 42:299-304. [PubMed: 9788407]

16. Newhauser W, Zheng Y, Taddei P, et al. Monte Carlo proton radiation therapy planning calculations. Trans Am Nuc Soc. 2008; 99:63-64.

17. Fontenot JD, Taddei $\mathrm{P}$, Zheng Y, et al. Organ equivalent dose and effective dose from stray radiation during passively scattered proton radiotherapy for prostate cancer. Phys Med Biol. 2008; 53:1677-1688. [PubMed: 18367796]

18. Zheng Y, Fontenot J, Taddei P, et al. Monte Carlo simulations of neutron spectral fluence, radiation weighting factor and ambient dose equivalent for a passively scattered proton therapy unit. Phys Med Biol. 2008; 53:187-201. [PubMed: 18182696]

19. Mutic S, Klein EE. A reduction in the AAPM TG-36 reported peripheral dose distributions with tertiary multileaf collimation. American Association of Physicists in Medicine Task Group 36. Int J Radiat Oncol Biol Phys. 1999; 44:947-953. [PubMed: 10386654]

20. Stovall M, Blackwell CR, Cundiff J, et al. Fetal dose from radiotherapy with photon beams: Report of AAPM Radiation Therapy Committee Task Group No. 36. Med Phys. 1995; 22:63-82. [PubMed: 7715571]

21. Howell RM, Hertel NE, Wang Z, et al. Calculation of effective dose from measurements of secondary neutron spectra and scattered photon dose from dynamic MLC IMRT for $6 \mathrm{MV}, 15$ MV, and 18 MV beam energies. Med Phys. 2006; 33:360-368. [PubMed: 16532941]

22. BEIR, VII. Health risks from exposure to low levels of ionizing radiation. National Research Council of the National Academies; Washington D.C.: 2006.

23. Hall EJ, Henry S. Kaplan Distinguished Scientist Award 2003. The crooked shall be made straight; dose-response relationships for carcinogenesis. Int J Radiat Biol. 2004; 80:327-337. [PubMed: 15223765]

24. Taddei P, Fontenot JD, Zheng Y, et al. Reducing stray radiation dose to patients receiving passively scattered proton radiotherapy for prostate cancer. Phys Med Biol. 2008; 53:2131-2147. [PubMed: 18369278]

25. Brenner DJ, Hall EJ. Secondary neutrons in clinical proton radiotherapy: A charged issue. Radiother Oncol. 2008; 86:165-170. [PubMed: 18192046]

26. Vargas C, Fryer A, Mahajan C, et al. Dose-volume comparison of proton therapy and intensitymodulated radiotherapy for prostate cancer. Int J Radiat Oncol Biol Phys. 2008; 70:744-751. [PubMed: 17904306]

27. Schneider U, Zwahlen D, Ross D, et al. Estimation of radiation-induced cancer from threedimensional dose distributions: Concept of organ equivalent dose. Int J Radiat Oncol Biol Phys. 2005; 61:1510-1515. [PubMed: 15817357]

28. Moyers M, Benton E, Ghebremedhin A, et al. Leakage and scatter radiation from a double scattering based proton beamline. Med Phys. 2008; 35:128-144. [PubMed: 18293570]

29. Wroe A, Rosenfeld A, Schulte R. Out-of-field dose equivalents delivered by proton therapy of prostate cancer. Med Phys. 2007; 34:3449-3456. [PubMed: 17926946]

30. Kellerer AM, Ruhm W, Walsh L. Indications of the neutron effect contribution in the solid cancer data of the A-bomb survivors. Health Phys. 2006; 90:554-564. [PubMed: 16691103] 


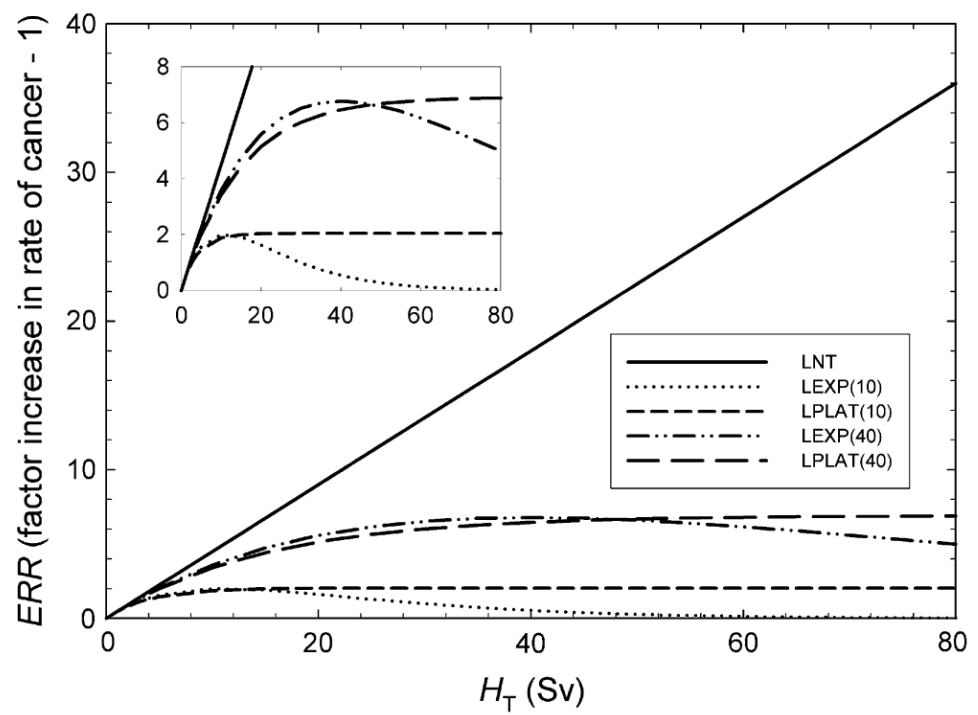

Fig. 1.

Excess relative risk $(E R R)$ as a function of equivalent dose $\left(H_{\mathrm{T}}\right)$. Differences in behavior reflect varying degrees of cell sterilization. Risk models include linear-no threshold (LNT); linear-exponential rolling off at $10 \mathrm{~Sv}$ [LEXP(10)]; linear-plateau rolling off at $10 \mathrm{~Sv}$ [LPLAT(10)]; linear-exponential rolling off at $40 \mathrm{~Sv}$ [LEXP(40)]; and linear-plateau rolling off at $40 \mathrm{~Sv}$ [LPLAT(40)]. 

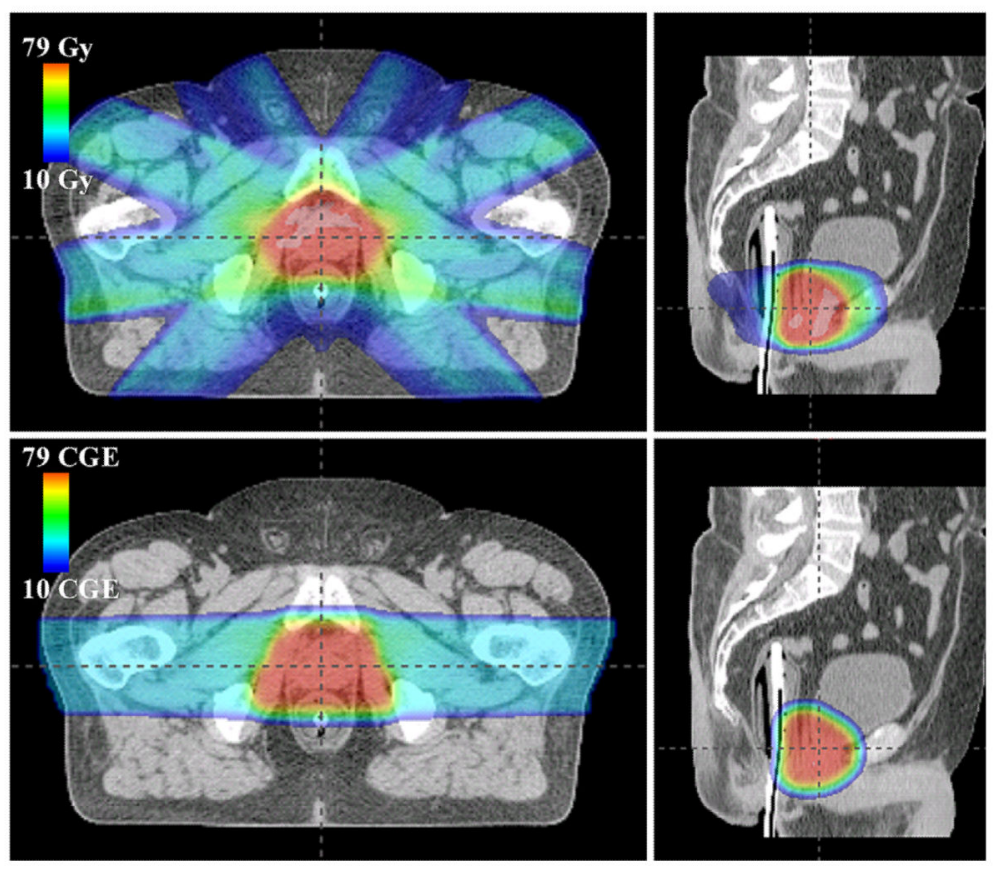

Fig. 2.

Axial (left) and sagittal (right) slices of absorbed dose distribution through isocentric plane for intensity-modulated X-ray therapy (top) and proton therapy (bottom) plans of the medium patient. $\mathrm{CGE}=$ cobalt gray equivalent. 


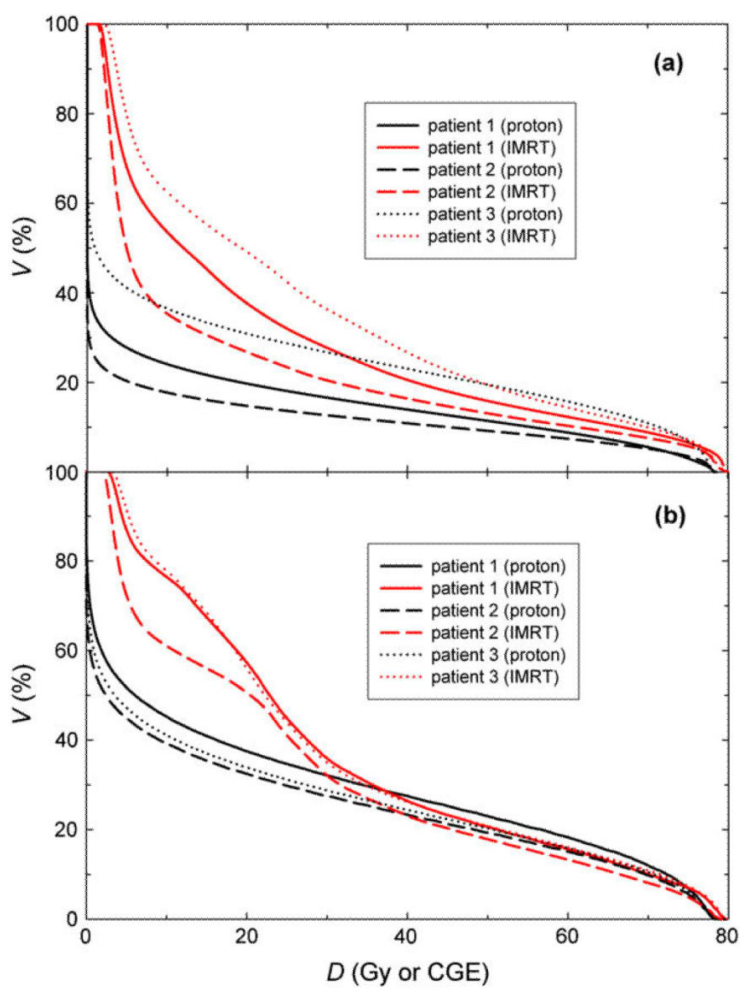

Fig. 3.

Dose $(D)$-volume $(V)$ histograms for the (a) bladder and (b) rectum of the patients studied. IMRT $=$ intensity-modulated X-ray therapy; CGE = cobalt gray equivalent. 


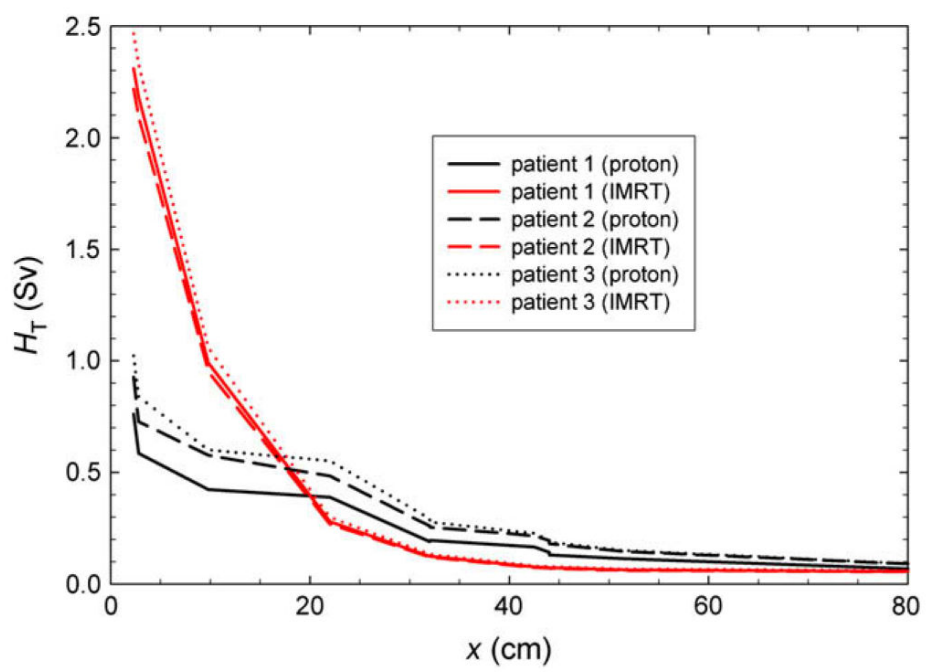

Fig. 4.

Equivalent dose $\left(H_{\mathrm{T}}\right)$ from scatter and leakage as a function of distance from the isocenter $(x)$ for the computed proton therapy and intensity-modulated X-ray therapy (IMRT) prostate plans. 


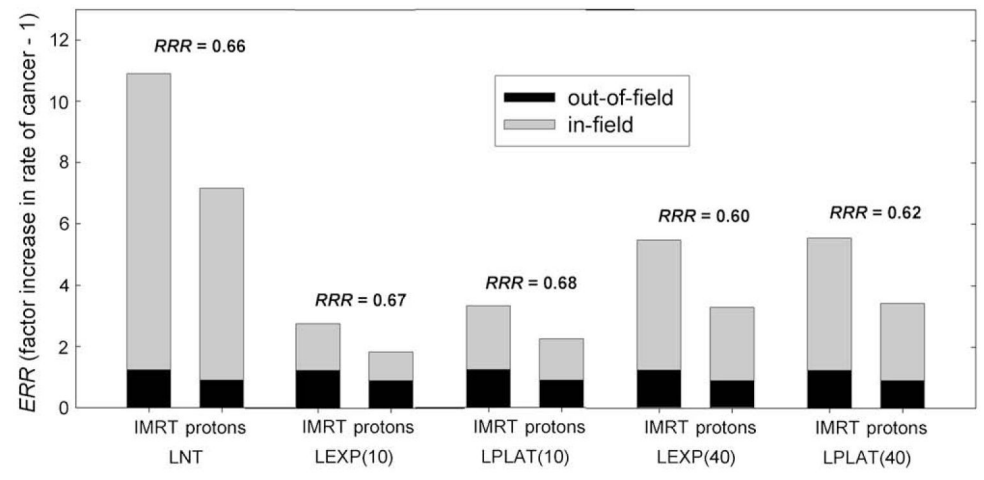

Fig. 5.

Excess relative risk $(E R R)$ of a secondary malignant neoplasm developing after intensitymodulated X-ray therapy (IMRT) and proton therapy for medium patient for various risk models. The quantity $R R R$ denotes the ratio of the $E R R$ from proton therapy to the $E R R$ from IMRT. Risk models include linear-no threshold (LNT); linear-exponential rolling off at 10 Sv [LEXP(10)]; linear-plateau rolling off at $10 \mathrm{~Sv}$ [LPLAT(10)]; linear-exponential rolling off at $40 \mathrm{~Sv}$ [LEXP(40)]; and linear-plateau rolling off at $40 \mathrm{~Sv}$ [LPLAT(40)]. 
Table 1

$R R R$ of SMN developing, defined as ratio of $E R R_{\text {proton }}$ to $E R R_{\mathrm{IMRT}}$, in 3 patients treated for prostate cancer

\begin{tabular}{|c|c|c|c|c|c|c|c|}
\hline \multirow[b]{3}{*}{ Organ } & \multicolumn{7}{|c|}{$E R R_{\mathrm{T}}$} \\
\hline & \multirow[b]{2}{*}{ ERR/Sv } & \multicolumn{2}{|c|}{ Patient 1 (small) } & \multicolumn{2}{|c|}{ Patient 2 (medium) } & \multicolumn{2}{|c|}{ Patient 3 (large) } \\
\hline & & Proton therapy & IMRT & Proton therapy & IMRT & Proton therapy & IMRT \\
\hline Colon & 0.51 & 2.53 & 3.13 & 2.25 & 2.73 & 2.39 & 3.15 \\
\hline Lung & 0.26 & 0.04 & 0.02 & 0.05 & 0.02 & 0.05 & 0.02 \\
\hline Stomach & 0.17 & 0.03 & 0.02 & 0.04 & 0.02 & 0.05 & 0.02 \\
\hline Bladder & 0.40 & 4.98 & 8.96 & 3.99 & 6.92 & 7.93 & 10.70 \\
\hline Liver & 0.26 & 0.05 & 0.03 & 0.07 & 0.03 & 0.07 & 0.03 \\
\hline Thyroid & 0.04 & 0.00 & 0.00 & 0.01 & 0.00 & 0.01 & 0.00 \\
\hline Bone marrow & 1.33 & 0.50 & 1.04 & 0.63 & 1.00 & 0.71 & 1.11 \\
\hline Remainder & 0.18 & 0.09 & 0.19 & 0.11 & 0.18 & 0.13 & 0.20 \\
\hline$E R R_{\text {modality }}$ & - & 8.2 & 13.4 & 7.1 & 10.9 & 11.3 & 15.2 \\
\hline$R R R$ & - & 0.61 & & 0.66 & & 0.74 & \\
\hline
\end{tabular}

Abbreviations: $R R R=$ ratio of excess relative risk; $\mathrm{SMN}=$ secondary malignant neoplasm; $E R R_{\text {proton }}=$ excess relative risk of a radiation-induced cancer developing after exposure to proton therapy; $E R R$ IMRT = excess relative risk of a radiation-induced cancer developing after exposure to intensity-modulated $\mathrm{X}$-ray therapy; $E R R \mathrm{~T}=$ excess relative risk of a radiation-induced cancer developing in tissue $T$; IMRT $=$ intensity-modulated $\mathrm{X}$-ray therapy; $E R R_{\mathrm{modality}}=$ excess relative risk for each modality (proton therapy or IMRT). 